\title{
Giant aneurysm of the bilateral vertebrobasilar junction treated by pipeline and coils: A case report and literature review
}

\author{
CHUN-LEI ZHANG ${ }^{1 *}$, ZHONG-HUA SHI $^{1 *}$, ZHI-ZHONG YAN $^{1}$, CHUN-LONG DING $^{1}$, \\ JIA-MING CAO $^{1}$, YU-HAI WANG ${ }^{1}$ and PENG ZHANG ${ }^{2}$ \\ ${ }^{1}$ Department of Neurosurgery, 904th Hospital of Chinese People's Liberation Army Joint Logistic Support, Wuxi, \\ Jiangsu 214004; ${ }^{2}$ Department of Neurosurgery, Xuanwu Hospital of Capital Medical University, Beijing 100053, P.R. China
}

Received October 29, 2019; Accepted July 14, 2020

DOI: $10.3892 / \mathrm{etm} .2020 .9472$

\begin{abstract}
Giant aneurysm of the posterior circulation is associated with a higher risk of rupture compared with that of the anterior circulation. Furthermore, surgical clipping and interventional embolization for giant aneurysm of the posterior circulation are more difficult and complex to perform. The present study reported on the case of a 26-year-old female who exhibited a giant spherical aneurysm of the vertebrobasilar junction (VBJ) with a maximum diameter of $\sim 35 \mathrm{~mm}$ that caused cervical discomfort. In addition, the patient experienced symptoms including left-sided walking and hoarseness caused by the compression of the brainstem and the posterior cranial nerves. The risks associated with performing surgery in this area are high and the prognosis is mainly poor. The patient of the present study was treated using the Pipeline Flex device with coil embolization. As a giant aneurysm of the VBJ simultaneously affects the bilateral vertebral arteries (VAs) and basilar artery, it is a unique condition and the treatment strategy must be personalized. Based on an analysis of the hemodynamic influence on the aneurysm in the present case, the Pipeline was placed through the left VA, the coils were packed through the right VA, and finally, the right VA was proximally occluded. At 7 months after embolization, the patient's modified Rankin scale score was 1 point. Upon analysis of the hemodynamic influence on the aneurysm of the VBJ, the VA with the larger
\end{abstract}

Correspondence to: Dr Yu-Hai Wang, Department of Neurosurgery, 904th Hospital of Chinese People's Liberation Army Joint Logistic Support, 101 Xingyuan North Road, Wuxi, Jiangsu 214004, P.R. China

E-mail: wangyuhai67@126.com

Dr Peng Zhang, Department of Neurosurgery, Xuanwu Hospital of Capital Medical University, 45 Changchun Street, Xicheng, Beijing 100053, P.R. China

E-mail: zhangpengwr@126.com

*Contributed equally

Key words: giant aneurysm, vertebrobasilar junction, endovascular treatment, pipeline embolization device shear force on the wall of the aneurysm was selected for occlusion to simplify the treatment of the aneurysm and to maximize the probability to achieve recovery.

\section{Introduction}

Giant intracranial aneurysm is a rare entity defined by a diameter of at least $25 \mathrm{~mm}$, accounting for $5 \%$ of all cases of intracranial aneurysm (1). Giant intracranial aneurysm of the posterior circulation occurs even more infrequently and is associated with high morbidity and mortality rates, even when treated. Due to the small size of the posterior fossa, unruptured giant posterior circulation aneurysm exerts a substantial mass effect on the brainstem and the adjacent cranial nerves, leading to cranial nerve palsy, motor impairment and hydrocephalus, and may result in variable levels of disability. Although the treatment of anterior circulation giant aneurysm with microsurgery and bypass has been moderately successful $(2,3)$, the treatment of posterior circulation giant aneurysm according to the same method is associated with poor prognosis, mainly due to occlusion of the perforating artery, resulting in brainstem ischemia (4). With regard to giant and complex intracranial aneurysm that cannot be managed by surgical strategies, Pipeline with coil embolization is an alternative treatment $(5,6)$. However, the mortality and morbidity rates of post-circulation aneurysm treated using flow diversion devices are much higher compared with those of pre-circulation aneurysm (7). In the present case report, contrast agent retention in the aneurysm lumen and the development of distal blood vessels were evaluated to determine the influence of bilateral vertebral artery blood flow dynamics on the aneurysm. Optimal treatment measures were then formulated in order to achieve the best possible recovery of the patient.

\section{Case report}

History and examination. The patient was a 26-year-old female admitted to Xuanwu Hospital of Capital Medical University (Beijing, China) in March 2018, who had been experiencing symptoms of neck discomfort for 1 year and left-sided walking for 1 month. In January 2017, the patient experienced neck discomfort, dizziness when raising the head after lowering it and a headache. The patient was first assumed 
to have cervical spondylosis and received physical therapy at a local hospital. However, the headache was not relieved. In February 2018, the patient experienced left-sided walking, left hand tremors, dysphagia and slight hoarseness. Local cervical MRI revealed no obvious signs of cervical disease; however, it did reveal a large, abnormal signal shadow associated with the vertebrobasilar artery. Further head MRI and magnetic resonance angiography (MRA) revealed a large vertebrobasilar aneurysm and obvious compression of the brain stem. Physical examination revealed an obvious left-slant tongue extension, hoarseness, left-hand tremors, Medical Research Council (8) grade 5 muscle strength in the left-limb and negative bilateral finger-to-nose and heel-to-knee tibia tests. After admission to the hospital, high-resolution MRI revealed a thrombus inside the aneurysm and an enhanced aneurysm wall (Fig. 1). The patient underwent digital subtraction angiography (DSA) at Xuanwu Hospital (Beijing, China) after 2 days of admission. A giant spherical aneurysm of the bilateral vertebrobasilar junction (VBJ) with a maximum diameter of $35 \mathrm{~mm}$ was identified during the surgery (Fig. 2). The neck of the aneurysm affected the bilateral vertebral arteries (VAs) and the basilar artery (BA). The right VA was more severely affected, and the bilateral posterior inferior cerebellar artery (PICA) remained at a distance from the aneurysm neck. Furthermore, the development of the posterior cerebral artery was not observed on right VA arteriography until the capillary phase (Fig. 3A), and the contrast agent was significantly delayed in the late venous phase (Fig. 3B); however, the development of the posterior cerebral artery was observed on left VA arteriography during the artery phase (Fig. 3C), and the contrast agent was slightly delayed in the same late venous phase (Fig. 3D).

Treatment strategy and course. Informed consent was provided by the patient and her relatives prior to endovascular therapy. A treatment plan was formulated after angiography. First, a microcatheter was placed through the right VA and the Pipeline was completely released through the left VA. Subsequently, the aneurysm was relatively tightly embolized with coils and the right VA was occluded near the aneurysm neck. Aspirin enteric-coated tablets (100 mg per day) and clopidogrel (75 mg per day) were administered for 6 days prior to treatment. At 9 days following hospitalization, the giant aneurysm of the VBJ was embolized using a Pipeline Flex device $(3.5 \times 25 \mathrm{~mm}$; Medtronic) and coils. A total of 32 coils were used for embolization, which included 10 Axium detachable 3D coils $(25 \mathrm{~mm}$ x $50 \mathrm{~cm}, \mathrm{x} 4 ; 22 \mathrm{~mm}$ x $50 \mathrm{~cm}, \mathrm{x} 3$; and $20 \mathrm{~mm}$ x $50 \mathrm{~cm}, \mathrm{x} 3$; Medtronic), 11 HydroFrame coils (18 mm x $50 \mathrm{~cm}, \mathrm{x} 3 ; 16 \mathrm{~mm}$ x $44 \mathrm{~cm}, \mathrm{x} 2 ; 14 \mathrm{~mm}$ x $45 \mathrm{~cm}, \mathrm{x} 2$; $13 \mathrm{~mm}$ x $47 \mathrm{~cm}, \mathrm{x} 2$; and $12 \mathrm{~mm}$ x $43 \mathrm{~cm}, \mathrm{x} 2$; MicroVention, Inc.), 11 Axium detachable Helix coils $(12 \mathrm{~mm} \mathrm{x} 40 \mathrm{~cm}, \mathrm{x} 2$; $10 \mathrm{~mm}$ x $30 \mathrm{~cm}, \mathrm{x} 4 ; 9 \mathrm{~mm}$ x $30 \mathrm{~cm}, \mathrm{x} 3$; and $8 \mathrm{~mm}$ x $30 \mathrm{~cm}$, $\mathrm{x} 2$; Medtronic). The final intraoperative angiography revealed that the aneurysm body did not develop and the contrast agent was slightly retained at the aneurysm neck (Fig. 4). For 2 days following the endovascular treatment, the patient was listless and complained of a headache worse than those that had occurred during the pre-embolization period. Physical examination revealed no new positive signs. Postoperative fluid administration of $3,000 \mathrm{ml}$ per day was performed until day 3 after the embolization, and subsequently, the patient was treated with a dexamethasone sodium phosphate injection at $10 \mathrm{mg}$ per day and injection of $125 \mathrm{ml} 20 \%$ mannitol every $8 \mathrm{~h}$ for a total of 7 days. After a further 2 days, the patient gradually recovered to the preoperative mental state. Re-examination of the head MRI and MRA on day 8 following endovascular treatment revealed a small residual aneurysm neck [Raymond-Roy classification, Class II (9)], and no new infarction in the posterior circulation area (Fig. 5). The patient was discharged on day 10 following embolization. The patient was prescribed dual antiplatelet therapy postoperatively, with clopidogrel ( $75 \mathrm{mg} /$ day) administered for 3 months and aspirin (100 mg/day) administered for 7 months from post-embolization day 1 .

Follow-up. A 3-month telephone follow-up revealed that the patient had increased white phlegm and drool and exhibited a slight choking cough when drinking water. Furthermore, the patient's left-limb muscle strength was slightly weakened compared with that pre-embolization and the patient required help from other individuals to walk. Three months after embolization, re-examination of the head MRI and MRA revealed that the aneurysm was completely occluded [Raymond-Roy classification, Class I (9)]; however, the brainstem was markedly compressed (Fig. 6), similar to the situation encountered during the pre-embolization stage. At 4 months after embolization, the levels of white phlegm and drool gradually decreased and the muscle strength of the left limb recovered. During the patient's second visit 7 months after the embolization, the patient presented with slow speech, small strides, no hoarseness in her voice, grade 1 in Kubota drinking test (10), grade 5 left-limb muscle strength, normal muscle tension and a negative bilateral finger-to-nose test. Re-examination of DSA revealed no aneurysm recurrence [Raymond-Roy classification, Class I (9)] and no stenosis in the parent artery (Fig. 7). In April 2020 (2 years after embolization), another video telephone follow-up was performed (Videos S1 and S2), which revealed that certain pre-embolization symptoms of the patient, including slow speech, small strides, hoarseness and choking cough while drinking water, were completely relieved. However, the patient still experienced weakness in both lower limbs after walking $>1,000$ meters. To date ( 2 years after the embolization), the modified Rankin scale score (11) of the patient is 1 point. The major symptoms and outcomes prior to pre-embolization, during the hospital stay and during the follow-up phase of the patient are summarized in Table I.

\section{Discussion}

Flow diverter devices (FDDs) are increasingly used in the treatment of intracranial aneurysm. A recent meta-analysis by Ye et al (7) reported an aneurysm occlusion rate of $78.8 \%$ after an average follow-up of 6.3 months, and neurological morbidity and mortality rates after FDD treatment were 9.8 and $3.8 \%$, respectively. The rupture rate of giant, unruptured, posterior circulation aneurysms within 5 years may be up to $50 \%$ (12). The relevant factors to be considered when treating such complex aneurysms include the shape, location, vascular architecture and collateral circulation of the aneurysm's parent artery. Treatment of these aneurysms has always posed a great challenge for neurosurgery. Microsurgery and 

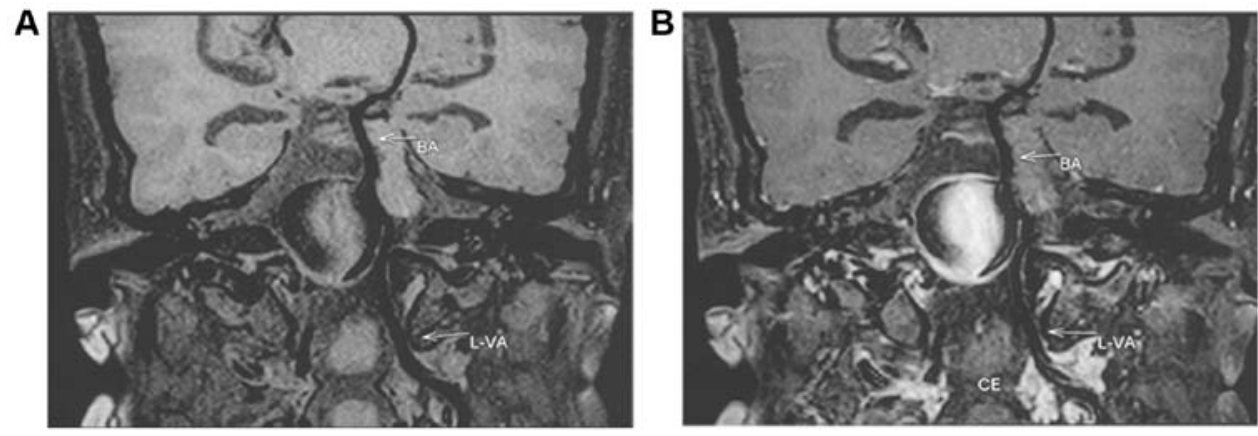

Figure 1. High-resolution MRI prior to embolization. (A) Plain scan image revealed a giant aneurysm at the vertebrobasilar junction. (B) An enhanced thrombus and wall of the aneurysm were observed in the enhanced image. BA, basilar artery; L-VA, left vertebral artery; CE, contrast enhancement.
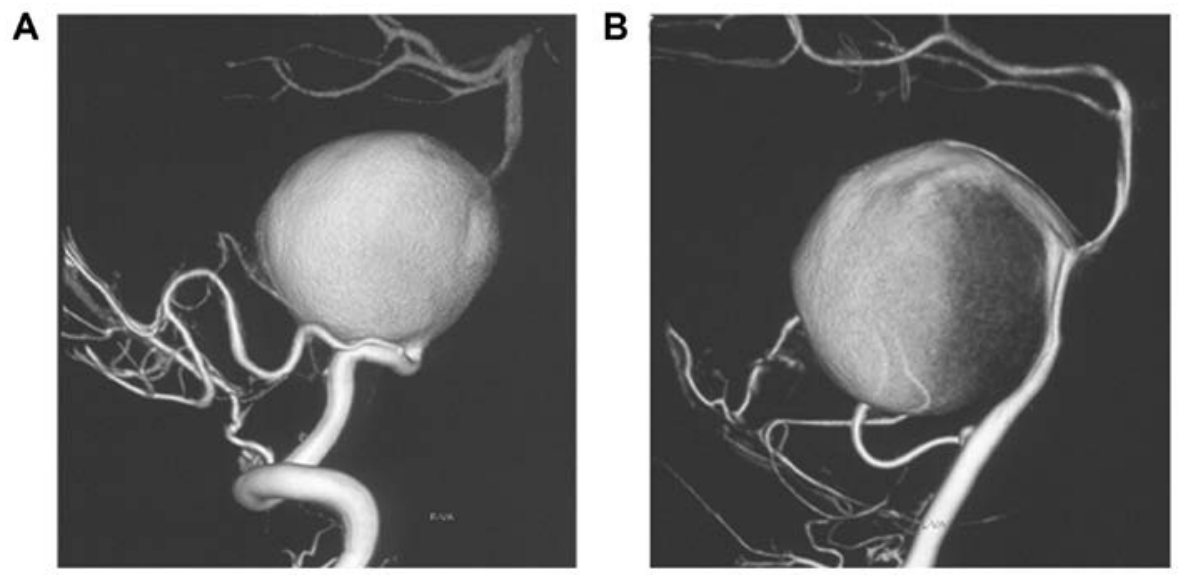

Figure 2. 3D-DSA prior to embolization. (A) 3D-DSA of the right VA. (B) 3D-DSA of the left VA. The bilateral posterior inferior cerebellar artery remained a short distance from the aneurysm neck. DSA, digital subtraction angiography; L/R-VA, left/right vertebral artery.

A

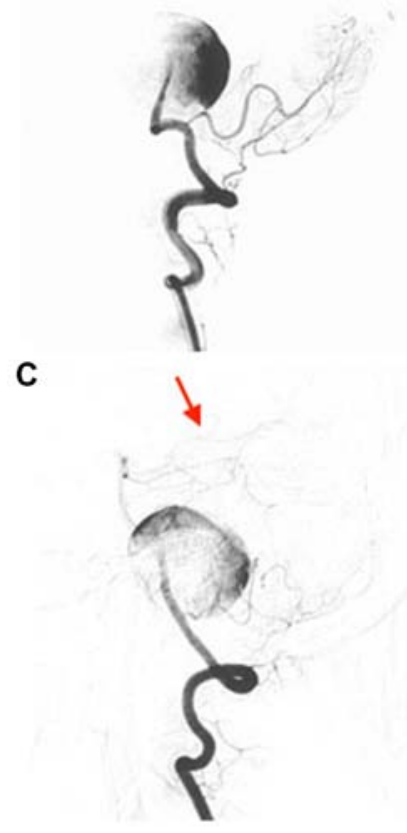

B

D

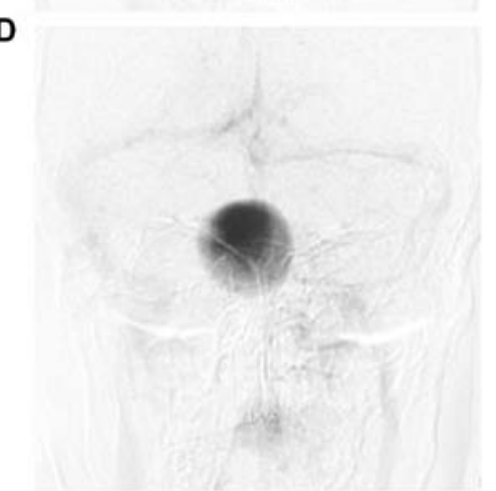

Figure 3. Angiography prior to embolization. (A) PCA was not observed on angiography of the right VA until the capillary phase. (B) The contrast agent (blue arrow) was significantly delayed in the late venous phase of the right VA. (C) PCA (red arrow) was observed on arteriography of the left VA only in the artery phase. (D) In the same late venous phase, contrast agent retention was lighter on angiography of the left VA than of the right VA. PCA, posterior cerebellar artery; VA, vertebral artery. 
A

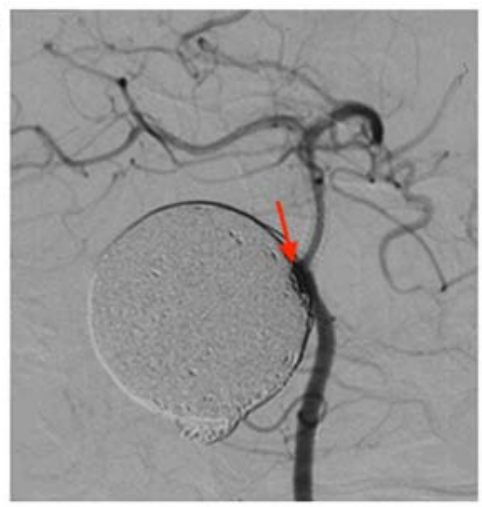

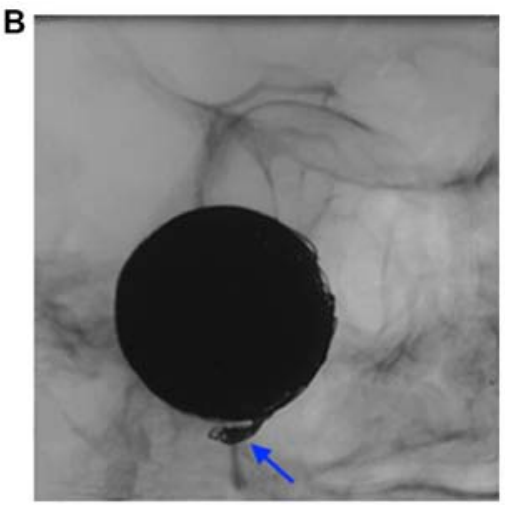

Figure 4. Immediate angiography following embolization. (A) The residual aneurysm neck was observed after embolization (red arrow) and (B) the right vertebral artery was occluded near the aneurysm neck (blue arrow).
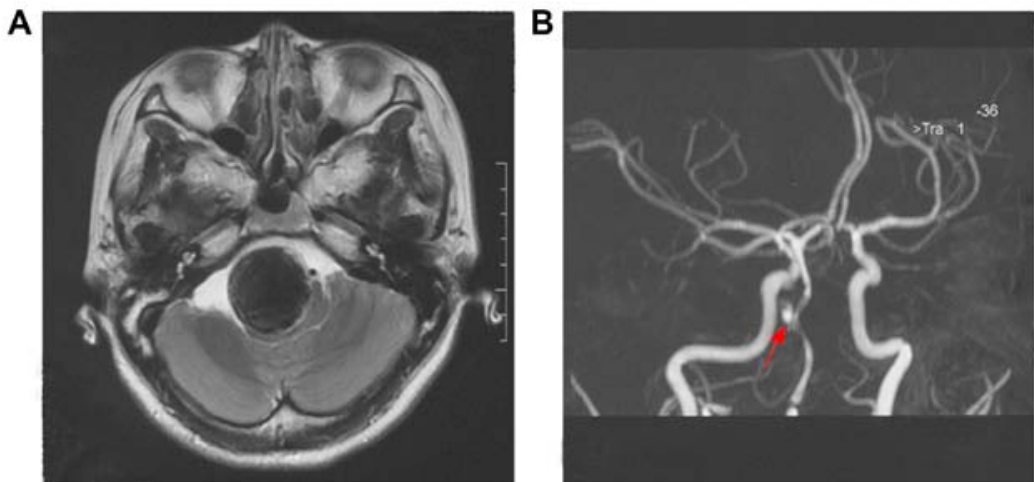

Figure 5. MRI on day 8 after embolization. (A) T2-weighted image revealed that the brain stem was still markedly compressed. (B) Magnetic resonance angiography revealed the residual aneurysm neck (red arrow).
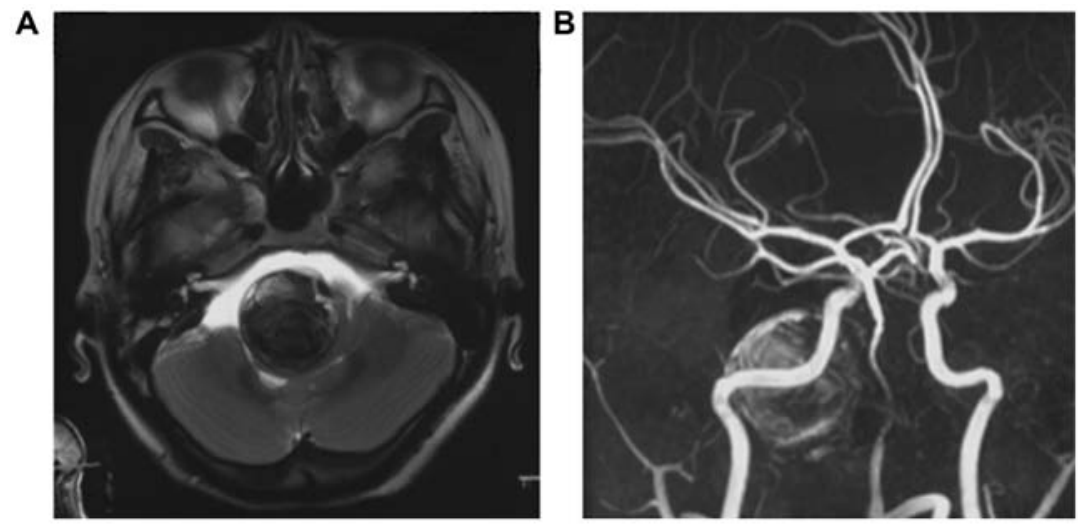

Figure 6. MRI at 3 months after embolization. (A) T2-weighted image revealed that the brain stem was still markedly compressed. (B) Magnetic resonance angiography revealed that the residual aneurysm neck had disappeared.

endovascular interventional therapy are associated with a high incidence of neurological complications $(4,7,13)$. Although the complication rate (the number of complications divided by the number of patients) of complex posterior circulation aneurysms treated with flow diversion devices is significantly higher compared with that of anterior circulation aneurysms, reconstruction of the parent artery with coil embolization is a suitable alternative when surgical treatment fails.

To date, only a small number of studies have reported on the treatment of giant aneurysm at the VBJ. Liang et al (14) described the endovascular treatment of 99 patients with large or giant aneurysms of the posterior circulation, 12 of which were located at the VBJ; 3 of those 12 cases were treated by occluding the contralateral VA with coils, and the bilateral VA was sacrificed in 1 of the 12 cases. However, in the latter case whose VA was sacrificed, the patient died 3 days after discharge from unknown causes. Furthermore, the prognosis of the 12 patients was not analyzed according to subgroups. In the present case study, the aneurysm neck simultaneously affected the bilateral VAs and the BA. The 
Table I. Major symptoms and outcomes for the patient prior to EM and during the hospital stay and the follow-up phase.

Follow-up after EM

\begin{tabular}{lcccccc}
\cline { 3 - 6 } Item & Pre-EM & In-hospital & 3 months & 4 months & 7 months & 2 years \\
\hline Dizziness & Moderate & Moderate & Mild & Disappeared & Disappeared & Disappeared \\
Headache & Mild & Mild & Disappeared & Disappeared & Disappeared & Disappeared \\
Hoarseness & Moderate & Moderate & Mild & Mild & Mild & Disappeared \\
Drinking of water (Kdt score) & 4 & 4 & 3 & 2 & 1 & 1 \\
Left-sided walking & Mild & Mild & Moderate & Mild & Disappeared & Disappeared \\
Left limb MS (MRC score) & $5-$ & 4 & $4-$ & $5-$ & 5 & 5 \\
mRS score & 2 & 2 & 4 & 2 & 1 & 1 \\
\hline
\end{tabular}

EM, embolization; FU, follow-up; Kdt, Kubota drinking test; MS, muscle strength; MRC, Medical Research Council; mRS, modified Rankin scale.
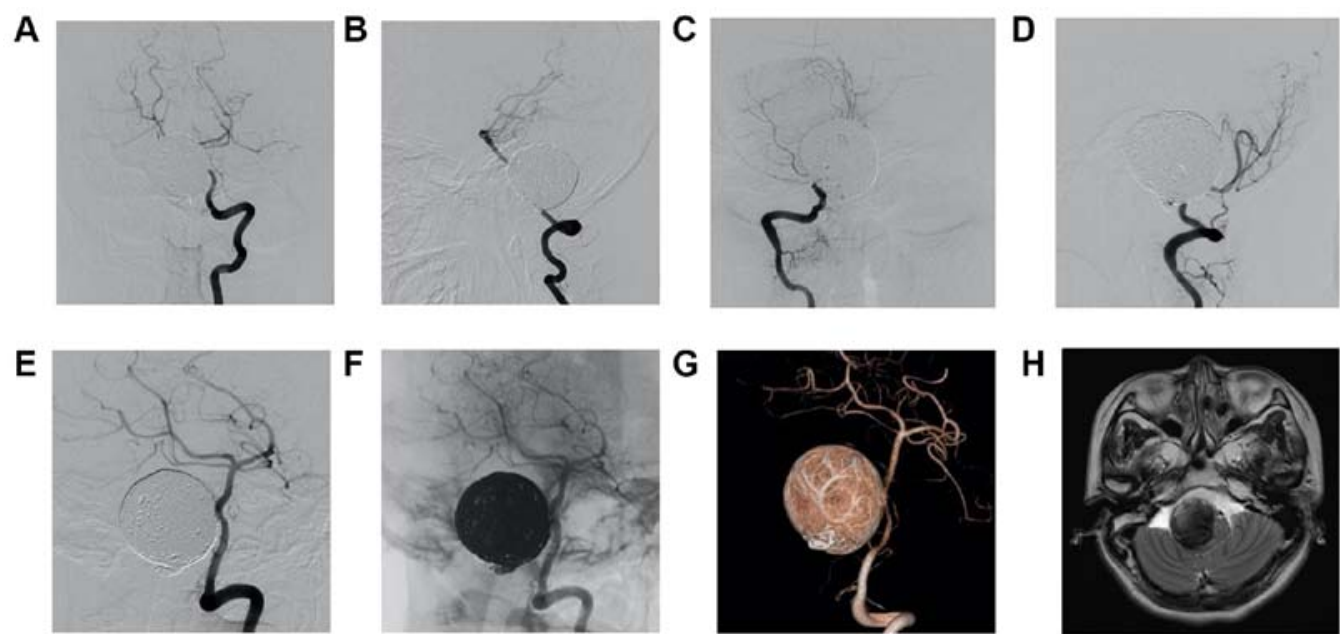

Figure 7. DSA and MRI at 7 months after embolization. The parent artery was unobstructed in the (A) anteroposterior and (B) lateral angiography images of the left VA. The left posterior inferior cerebellar artery was observed in the (C) anteroposterior and (D) lateral angiography images of the right VA. (E) No stenosis or residual aneurysm neck were observed on tangent position angiography of the left VA. (F) The spherical shape of the coils was not significantly changed. (G) 3-dimensional DSA of the left VA. (H) T2-weighted MRI revealed that the brain stem was still markedly compressed. VA, vertebral artery; DSA, digital subtraction angiography.

bilateral PICAs remained at a distance from the aneurysm neck and the development of the anterior spinal artery (ASA) was observed on angiography of the right VA; the right VA was associated with the development of ASA, which was near the aneurysm neck. In view of the hemodynamic complexity of this aneurysm, it was crucial to select a suitable type of treatment in order to reduce the hemodynamic effect on the aneurysm. On the basis of using the computational fluid dynamics method, Jing et al (15) discovered that adjunctive coiling with the placement of a Tubridge flow diverter may significantly reduce intra-aneurysmal flow velocity and wall shear stress, promoting thrombosis formation and occlusion of aneurysms. Xiang et al (16) suggested that the results of aneurysm occlusion may be predicted with high fidelity by calculating the intracavitary velocity, inflow rate and shear force on the wall prior to and after implantation of the flow device through computer simulation; however, factors associated with the vascular anatomy must also be considered. Given the hemodynamic particularity of the aneurysm in the present case, it was possible to select the VA on the side that exerted the greater influence on the hemodynamics of the aneurysm for occlusion, and the Pipeline was able to be placed through the VA on the other side. In the present case, the development of the posterior cerebral artery was not observed on right VA arteriography until the capillary phase and perfusion with the contrast agent was significantly delayed in the late venous phase; however, the development of the posterior cerebral artery was observed on left VA arteriography during the artery phase and perfusion with the contrast agent was slightly delayed in the same late venous phase. Therefore, the hemodynamics of the right VA may have a greater impact on aneurysms, although occluding the right VA may affect the ASA. Fortunately, the contralateral blood vessels of the patient were anastomosed with the right ASA. Therefore, it was possible to place the embolization microcatheter into the aneurysm cavity through the right VA and the Pipeline was subsequently completely released through the left VA. Furthermore, the aneurysm was relatively tightly embolized 
with coils and the right VA was finally occluded near the aneurysm neck.

Previous studies have indicated that the combination of coils and Pipeline for aneurysm treatment may increase the embolization rate and reduce the risk of bleeding $(17,18)$. Dense packing of coils may result in a mass effect, whereas loose packing may cause delayed rupture of the large aneurysm; therefore, it is important to consider carefully the extent to which coils should be packed. A single-center study (19) suggested that treatment with Pipeline and coils resulted in a higher embolization rate compared with Pipeline alone for aneurysms $<10 \mathrm{~mm}$. Peschillo et al (20) also reported that, for large and giant carotid aneurysms, treatment with flow-diverter stents in combination with coils may provide a higher aneurysm occlusion rate and reduce the requirement for retreatment compared with the use of flow-diverter stents alone. However, to date, there is no objective method for determining the appropriate degree of packing coils for giant aneurysms. Autopsy and histopathological analysis performed on a patient who died due to delayed rupture of aneurysm 34 days after treatment with Pipeline and coils suggested that the most relevant contributing factor in aneurysm rupture was the lack of thrombus formation in the inflow area, and thrombus coverage in the outflow area resulted in increased pressure of the aneurysm (21). Therefore, it may be more important to reduce the hemodynamic impact on aneurysms by performing relatively dense embolization for rapid thrombolysis, since the prognosis is poor following delayed rupture.

Insufficient aneurysm management occurred in the present case when MRI on day 8 after embolization revealed that the residual aneurysm neck and mass effect were difficult to treat. Residual aneurysm neck in a giant aneurysm may increase the risk of delayed rupture (21). In the present case, a novel treatment strategy was implemented, which may lead to improved outcomes through minimizing the flow to the aneurysm by occlusion of the right VA according to hemodynamics and the guidance of blood flow by the Pipeline to accelerate the thrombolysis and endothelialization of the aneurysm neck (14), a procedure that may promote the complete embolization of aneurysms. Upon analysis of the hemodynamic influence on the aneurysm of the VBJ, the VA with the larger shear force on the wall of the aneurysm was selected for occlusion to simplify the treatment of the aneurysm and to maximize the probability to achieve recovery, which may be more effective to treat similar aneurysms.

\section{Acknowledgements}

Not applicable.

\section{Funding}

No funding was received.

\section{Availability of data and methods}

The datasets used and/or analyzed during the present study are available from the corresponding author on reasonable request.

\section{Authors' contributions}

CLZ and ZHS made substantial contributions to the design of the study. YHW and PZ made substantial contributions to the conception and design of the study, and they also contributed in drafting the manuscript and revising it critically for important intellectual content, as well as in the collection of data. CLZ made substantial contributions to the conception of the study, as well as the acquisition, analysis and interpretation of data. ZZY, CLD and JMC were involved in the acquisition of data. CLZ and ZHS contributed in drafting the manuscript and revising it critically for important intellectual content. All authors read anad approved the final manuscript.

\section{Ethics approval and consent to participate}

The present study was approved by the Medical Ethics Committee of the 904th Hospital of the People's Liberation Army (Wuxi, China) and Xuanwu Hospital of Capital Medical University (Beijing, China). Written informed consent was obtained from the participant.

\section{Patient consent for publication}

The patient provided consent for publication.

\section{Competing interests}

The authors declare that they have no competing interests.

\section{References}

1. Haemmerli J, Lenga P, Hong B, Kursumovic A, Maldaner N, Burkhardt JK, Bijlenga P, Rüfenacht DA, Schmidt NO, Vajkoczy P and Dengler J: Clinical implications and radiographic characteristics of the relation between giant intracranial aneurysms of the posterior circulation and the brainstem. Acta Neurochir (Wien) 161: 1747-1753, 2019.

2. Luzzi S, Gallieni M, Del Maestro M, Trovarelli D, Ricci A and Galzio R: Giant and very large intracranial aneurysms: Surgical strategies and special issues. Acta Neurochir Suppl 129: 25-31, 2018.

3. Chen J, Zhang C, Li P, Chen L and Wang Y: High-flow extracranial-intracranial bypass for giant cavernous carotid aneurysm. J Craniofac Surg 29: 1042-1046, 2018.

4. Ota N, Matsukawa H, Noda K, Sato H, Hatano Y, Hashimoto A, Miyazaki T, Kondo T, Kinoshita Y, Saito N, et al: Evaluation of microsurgery for managing giant or complex cerebral aneurysms: A retrospective study. World Neusurg 115: e190-e199, 2018.

5. Griessenauer CJ, Ogilvy CS, Adeeb N, Dmytriw AA, Foreman PM, Shallwani H, Limbucci N, Mangiaflco S, Kumar A, Michelozzi C, et al: Pipeline embolization of posterior circulation aneurysms: A multicenter study of 131 aneurysms. J Neurosurg 130: 923-935, 2018.

6. Walcott BP, Stapleton CJ, Choudhri O and Patel AB: Flow diversion for the treatment of intracranial aneurysms. JAMA Neurol 73: 1002-1008, 2016.

7. Ye G, Zhang M, Deng L, Chen X and Wang Y: Meta-analysis of the efficiency and prognosis of intracranial aneurysm treated with flow diverter devices. J Mol Neurosci 59: 158-167, 2016.

8. Gregson JM, Leathley MJ, Moore AP, Smith TL, Sharma AK and Watkins CL: Reliability of measurements of muscle tone and muscle power in stroke patients. Age Ageing 29: 223-228, 2000.

9. Raymond J, Guilbert F, Weill A, Georganos SA, Juravsky L, Lambert A, Lamoureux J, Chagnon M and Roy D: Long-term angiographic recurrences after selective endovascular treatment of aneurysms with detachable coils. Stroke 34: 1398-1403, 2003.

10. Wu MC, Chang YC, Wang TG and Lin LC: Evaluating swallowing dysfunction using a $100-\mathrm{ml}$ water swallowing test. Dysphagia 19: 43-47, 2004. 
11. van Swieten JC, Koudstaal PJ, Visser MC, Schouten HJ and van Gijn J: Interobserver agreement for the assessment of handicap in stroke patients. Stroke 19: 604-607, 1988.

12. Wiebers DO, Whisnant JP, Huston J III, Meissner I, Brown RD Jr, Piepgras DG, Forbes GS, Thielen K, Nichols D, O'Fallon WM, et al: Unruptured intracranial aneurysms: Natural history, clinical outcome, and risks of surgical and endovascular treatment. Lancet 362: 103-110, 2003.

13. GrazianoF,Ganau M,Iacopino DG and BoccardiE: Vertebro-basilar junction aneurysms: A single centre experience and meta-analysis of endovascular treatments. Neuroradiol J 27: 732-741, 2014.

14. Liang F, Zhang Y, Yan P, Ma C, Liang S, Jiang P and Jiang C: Predictors of periprocedural complications and angiographic outcomes of endovascular therapy for large and giant intracranial posterior circulation aneurysms. World Neurosurg 125: e378-e384, 2019.

15. Jing L, Zhong J, Liu J, Yang X, Paliwal N, Meng H, Wang S and Zhang Y: Hemodynamic effect of flow diverter and coils in treatment of large and giant intracranial aneurysms. World Neurosurg 89: 199-207, 2016.

16. Xiang J, Damiano RJ, Lin N, Snyder KV, Siddiqui AH, Levy EI and Meng H: High-fidelity virtual stenting: Modeling of flow diverter deployment for hemodynamic characterization of complex intracranial aneurysms. J Neurosurg 123: 832-840, 2015.

17. Lubicz B, Collignon L, Raphaeli G, Pruvo JP, Bruneau M, De Witte $\mathrm{O}$ and Leclerc $\mathrm{X}$ : Flow-diverter stent for the endovascular treatment of intracranial aneurysms: A prospective study in 29 patients with 34 aneurysms. Stroke 41: 2247-2253, 2010.
18. Siddiqui AH, Kan P, Abla AA, Hopkins LN and Levy EI: Complications after treatment with pipeline embolization for giant distal intracranial aneurysms with or without coil embolization. Neurosurgery 71: E509-E513, 2012.

19. Lin LM, Colby GP, Kim JE, Huang J, Tamargo RJ and Coon AL: Immediate and follow-up results for 44 consecutive cases of small $(<10 \mathrm{~mm})$ internal carotid artery aneurysms treated with the pipeline embolization device. Surg Neurol Int 4: 114, 2013.

20. Peschillo S, Caporlingua A, Resta MC, Peluso JPP, Burdi N, Sourour N, Diana F, Guidetti G, Clarençon F, Bloemsma GC, et al: Endovascular treatment of large and giant carotid aneurysms with flow-diverter stents alone or in combination with coils: A multicenter experience and long-term follow-up. Oper Neurosurg (Hagerstown) 13: 492-502, 2017.

21. Ikeda H, Lshii A, Kikuchi T, Ando M, Chihara H, Arai D, Hattori E and Miyamoto S: Delayed aneurysm rupture due to residual blood flow at the inflow zone of the intracranial paraclinoid internal carotid aneurysm treated with the pipeline embolization device: Histopathological investigation. Interv Neuroradiol 21: 674-683, 2015.

This work is licensed under a Creative Commons Attribution-NonCommercial-NoDerivatives 4.0 International (CC BY-NC-ND 4.0) License. 